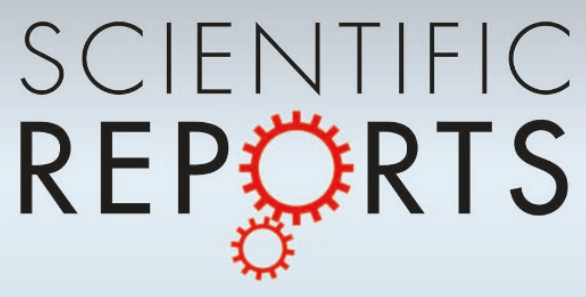

OPEN

SUBJECT AREAS:

PAEDIATRIC RESEARCH

HAEMATOLOGICAL CANCER

Received

1 August 2014

Accepted

20 October 2014

Published

6 November 2014

Correspondence and requests for materials should be addressed to

Y.N. (yxnakaza@ shinshu-u.ac.jp)

\section{Reduced-toxicity myeloablative} conditioning consisting of 8-Gy total body irradiation, cyclophosphamide and fludarabine for pediatric hematological malignancies

Koichi Hirabayashi, Yozo Nakazawa, Kazuo Sakashita, Takashi Kurata, Shoji Saito, Kentaro Yoshikawa, Miyuki Tanaka, Ryu Yanagisawa \& Kenichi Koike

Department of Pediatrics, Shinshu University School of Medicine, Matsumoto, Japan.

Conventional myeloablative conditioning (MAC) regimens often cause severe regimen-related toxicity (RRT). Furthermore, many patients suffer from poor quality of life in accordance with the increase in long-term survivors. We therefore devised a reduced-toxicity myeloablative conditioning (RTMAC) regimen consisting of 8-Gy total body irradiation (TBI), fludarabine (FLU) and cyclophosphamide (CY) for pediatric hematological malignancies. A retrospective single-center analysis was performed on patients with leukemia or myelodysplastic syndrome (MDS), aged $\leq 20$ years, who had received an 8 -Gy TBI/FLU/CY RTMAC regimen followed by allogeneic hematopoietic stem cell transplantation (allo-HSCT). Thirty-one patients underwent first allo-HSCT after an RTMAC regimen. The diagnoses were acute lymphoblastic leukemia $(n=11)$, acute myeloid leukemia $(n=13)$, $\operatorname{MDS}(n=4)$, juvenile myelomonocytic leukemia $(n=$ 1) and acute leukemias of ambiguous lineage $(n=2)$. While 3 patients showed early hematological relapse, the remaining 28 patients achieved engraftments. None of the patients developed grade 4 or 5 toxicities during the study period. The 5 -year overall survival and relapse-free survival were $80 \%$ [95\% confidence interval: CI, $61-91 \%]$ and $71 \%$ [95\% CI, 52-84\%], respectively. Our RTMAC regimen would be less toxic and offers a high probability of survival for children with hematological malignancies.

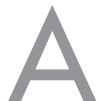

llogeneic hematopoietic stem cell transplantation (allo-HSCT) is a standard treatment for children with high-risk acute myeloid leukemia (AML) and very high-risk acute lymphoblastic leukemia (ALL) ${ }^{1,2}$. The antileukemic efficacy of allo-HSCT is attributed to high-dose chemotherapy with or without radiotherapy, and graft-versus-leukemia (GVL) effects mediated by donor immune cells ${ }^{3}$. The clinical significance of allo-HSCT has long been based on the assumption that myeloablative doses of cytotoxic therapy are requisite for both disease eradication and host immunosuppression ${ }^{4}$. Conventional myeloablative conditioning (MAC) regimens, however, often cause severe regimen-related toxicity (RRT). Accordingly, elderly patients or patients with poor performance status (PS) are not able to receive allo-HSCT combined with conventional MAC regimens because of their direct adverse effects on various organs. In addition, RRT may impede the effective delivery of drugs for the prophylaxis/treatment of infections and for graft-versus-host disease (GVHD) $)^{5,6}$.

To reduce MAC-induced toxicity, reduced-intensity conditioning (RIC) and non-myeloablative conditioning (NMAC) were introduced to adult patients from $1995^{7}$. This idea was driven from findings that the curative potential of allo-HSCT is not only due to the intensity of conditioning, but also to its immunologic GVL effects. NMAC/RIC was mainly used for adult patients with AML, myelodysplastic syndrome (MDS) or chronic myeloid leukemia, for which GVL effects are expected. On the other hand, NMAC/RIC is applied to a small number of ALL patients because of inadequate GVL effects and poor outcome ${ }^{8-10}$. Recently, a novel reduced-toxicity myeloablative conditioning (RTMAC) regimen, i.e., the combination of fludarabine (FLU) and busulfan (BU), was shown to provide better outcome, when compared with NMAC/RIC, for adult leukemia ${ }^{11,12}$.

For childhood hematological malignancies, conventional MAC regimens have been continuously used as the preferred conditioning, because more intensive pretransplant approaches are tolerable for pediatric patients with 
good PS. Nevertheless, many patients suffer from poor quality of life in accordance with the increase in long-term survivors who received allo-HSCT after a conventional MAC regimen during childhood ${ }^{13-17}$. We therefore devised an RTMAC regimen consisting of 8-Gy total body irradiation (TBI), FLU and cyclophosphamide (CY) for pediatric hematological malignancies, and reported low toxicity during the early post-transplantation period ${ }^{18}$. Since median follow-up period has exceeded 7 years, we here report the details of outcomes, efficacy and safety.

\section{Results}

Patients and disease characteristics. The characteristics of the 31 patients who underwent first allo-HSCT after an 8-Gy TBI/FLU/CY regimen are summarized in Table 1 . The diagnoses were ALL $(n=$ $11), \operatorname{AML}(n=13), \operatorname{MDS}(n=4), \operatorname{JMML}(n=1)$ and acute leukemias of ambiguous lineage (ALAL) $(n=2)$. The median age of patients was 8.0 years (range, $0.8-18.7$ years). Fifteen patients received bone marrow transplantation (BMT) from related donors. Six patients underwent unrelated BMT. The remaining 10 patients received unrelated cord blood transplantation (CBT). Before allo-HSCT, all of the 31 patients were at grade 0 or 1 of the Eastern Cooperative Oncology Group Performance Status grading. Two patients who had central nervous system disease received additional craniospinal irradiation after allo-HSCT.

Engraftment. While 3 patients showed early hematological relapse, the remaining 28 patients achieved neutrophil and platelet engraftments after the median of 19 days (range, 9-42 days) and 31 days (range, 14-99 days), respectively. Full donor chimerism was obtained within 100 days in all of these 28 patients (Table 2).

Toxicities. RRT at grades of 1, 2 and 3 appeared in 21 (16 patients, diarrhea; 3 patients, cytomegalovirus reactivation; 2 patients, mucositis oral), 6 (5 patients, diarrhea; 1 patient, mucositis oral) and 10 (3 patients, hemorrhagic cystitis; 2 patients, catheter-related infection; 2 patients, sepsis, 1 patient, pancreatitis; 1 patient, fungal pneumonia; 1 patient, mucositis oral) of the patients, respectively. Furthermore, none of the patients developed grade 4 or 5 toxicities during the study period in patients who did not relapse after first alloHSCT.

GVHD. Incidences of acute and chronic GVHD were evaluated in the 28 patients who achieved engraftment (Table 2). Twelve patients experienced grade III acute GVHD, but grade IV was not found. Moderate to severe chronic GVHD occurred in 4 of the 28 patients. The median times of onset of acute and chronic GVHD were 21 days (range, 10-55 days) and 4 months (range, 3-12 months), respectively.

Survival. The median follow-up period was 7.7 years (range, 2.410.5 years). Twenty-four patients are disease-free alive at present. Nine patients ( 2 patients with ALL and 7 patients with AML) relapsed after the first allo-HSCT. The median time of onset of relapse after allo-HSCT for the 9 patients who relapsed was 246 days (range, 9-722 days). Six patients with relapsed leukemia/MDS (2 patients, ALL; 3 patients, AML; 1 patient, MDS) received a second allo-HSCT after a preparative regimen consisting of cytarabine, idarubicin and FLU for 3 patients, after a full dose BU-based regimen for 2 patients, and after an 8-Gy TBI/FLU/CY regimen for 1 patient. The median interval between first allo-HSCT and second allo-HSCT was 16 months (range, 1.5-24 months). After the second allo-HSCT, 2 AML patients have survived without leukemic relapse. As presented in Figure 1, the 5-year overall survival (OS) and relapsefree survival (RFS) of a total of 31 patients transplanted after an RTMAC regimen were $80 \%$ (95\% confidence interval: CI, 61-91\%) and $71 \%$ (95\% CI, 52-84\%), respectively. The 5-year OS and RFS of

Table 1 | Characteristics of 31 patients who received allo-HSCT after 8-Gy TBI/FLU/CY conditioning regimen

Characteristics Value

Sex, no. Male
Female
Median age at transplantation, years
(range)
Median time from diagnosis to
transplantation, months (range)
Diagnoses, no. of recipients
ALL

16

15

$8.0(0.8-18.7)$

9 (2-92)

ALL

CR $1(M R D+)$

CR2 (MRD+)

B-lineage (MLL rearrangement)

T-lineage

AML

CR 1

CR2

Refractory disease

M2 [t(8;21)]

M3 $[+(15 ; 17]$

M5 (MLL rearrangement)

M6

M7 (monosomy 7/trisomy 8)

MDS

CR 1

Untreated primary disease

RAEB-2 (monosomy 7)

RCMD (monosomy 7)

JMML

Untreated primary disease

ALAL

CR 1 (MRD+)

CR2 (MRD+)

Cytogenetic abnormalities

MLL rearragement

monosomy 7

trisomy 8

$\mathrm{t}(8 ; 21)$

$\mathrm{t}(15 ; 17)$

normal karyotype

others

Central nervous system disease

Stem cell source (HLA-matching: A, B, C, DRB I)

Related BM, 8/8

Related BM, 7/8

Related BM, 6/8

Related BM, $5 / 8$

Related BM, $4 / 8$

Unrelated BM, 8/8

Unrelated $\mathrm{BM}, 7 / 8$

Unrelated CB, 7/8

Unrelated $C B, 6 / 8$

Unrelated CB, $5 / 8$

Infused cell dose

BM TNC, $\times 10^{8}$

$\mathrm{BMCD} 34, \times 10^{6}$

CB TNC, $\times 10^{8}$

$\mathrm{CB} C D 34, \times 10^{6}$

11

$4(0)$

7 (0)

$9(2)$

2

13

3

4

$5(4)$

1 (1)

1 (1)

$5(2 / 3)$

4

1

3

2 (1)

2 (2)

1

1 (0)

1 (0)

3

5

3

10

5

2

6

3

2

1

3

4

2

4

5

$4.30(1.9-15.6)$

$3.99(1.8-14.0)$

$0.65(0.23-1.26)$

0.14 (0.07-0.27)

ALAL, acute leukemias of ambiguous lineage; $A L L$, acute lymphoblastic leukemia; $A M L$, acute myeloid leukemia; $B M$, bone marrow; $C B$, cord blood; $C R$, complete remission; $C Y$, cyclophosphamide; FLU, fludarabine; HLA, human leukocyte antigen; HSCT, hematopoietic stem cell transplantation; JMML, juvenile myelomonocytic leukemia; MDS, myelodysplastic syndrome; MRD+, minimal residual disease positive; RAEB, refractory anemia with excess blasts; RCMD, refractory cytopenia with
dition multilineage dysplasia; TBI, total body irradiation; TNC, total nucleated cells.

11 ALL patients were 91\% (95\% CI, 51-99\%) and 82\% (95\% CI, $45-$ $95 \%)$, respectively. The 5-year OS and RFS of 18 patients with myeloid malignancies (AML, MDS and JMML) were 71\% (95\% CI, $43-87 \%)$ and $61 \%$ (95\% CI, 35-79\%), respectively. 


\begin{tabular}{|c|c|c|c|}
\hline & BMT & CBT & Total \\
\hline \multicolumn{4}{|l|}{$\begin{array}{l}\text { No. of patients } \\
\text { Engraftment }\end{array}$} \\
\hline Neutrophil engraftment & 19 & 9 & 28 \\
\hline Median interval of neutrophil engraftment, days (range) & $17(9-28)$ & $30(18-42)$ & $19(9-42)$ \\
\hline Platelet engraftment & & & \\
\hline & \multicolumn{2}{|c|}{ Chimerism/relapse up to day 100} & 31 (14-99) \\
\hline Full chimerism & 19 & 9 & 28 \\
\hline Mixed chimerism without early relapse & 0 & 0 & 0 \\
\hline \multirow{2}{*}{\multicolumn{4}{|c|}{ Acute GVHD }} \\
\hline & & & \\
\hline Grade 0 & 6 & 3 & 9 \\
\hline Grade I & 5 & 1 & 6 \\
\hline Grade II & 0 & 1 & 1 \\
\hline Grade III & 8 & 4 & 12 \\
\hline Grade IV & 0 & 0 & 0 \\
\hline Not assessable & 2 & 1 & 3 \\
\hline \multicolumn{4}{|l|}{ Chronic GVHD } \\
\hline None & 12 & 9 & 21 \\
\hline Mild & 3 & 0 & 3 \\
\hline Moderate & 3 & 0 & 3 \\
\hline Severe & 1 & 0 & 1 \\
\hline Not assessable & 2 & 1 & 3 \\
\hline
\end{tabular}

BMT, bone marrow transplantation; CBT, cord blood transplantation; CY, cyclophosphamide; FLU, fludarabine; GVHD, graft-versus-host disease; HSCT, hematopoietic stem cell transplantation; TBI, total body irradiation.

Prognostic factors for survival. (Table 3) We evaluated prognostic factors in the 31 patients who received allo-HSCT after 8-Gy TBI/ FLU/CY regimen. Univariate analysis showed no effects from age, sex, stem cell source, human leukocyte antigen (HLA) compatibility, GVHD and interval from onset to first allo-HSCT on 5-year OS and RFS. Only the disease status [non-complete remission (non-CR) vs. $\mathrm{CR}$ ] had a significant impact on both 5 -year OS and RFS $(p<0.01$ and 0.03 , respectively).

\section{Discussion}

NMAC/RIC have been reported as the preparative regimen for alloHSCT of pediatric patients with hematological malignancy in poor PS and for second allo-HSCT ${ }^{19-24}$. In these days, non TBI-based RTMAC regimens have been used for first allo-HSCT of pediatric patients with hematological malignancy ${ }^{25-27}$. On the other hand, there is only one report on TBI-based RTMAC regimen (9-Gy TBI + FLU + melphalan) for pediatric hematological malignancies ${ }^{28}$. The aim of this study was to evaluate the efficacy of the 8-Gy TBI/ FLU/CY regimen that was used as a preparative conditioning of first allo-HSCT for pediatric patients with hematological malignancies who had a good PS.

There are some favorable outcomes in allo-HSCT after our RTMAC regimen; (1) free from non-relapse mortality (NRM), (2) low frequency of graft failure, especially in CBT, (3) feasibility of second allo-HSCT after relapse.

(1) In pediatric allo-HSCT, the cumulative incidences of NRM after conventional MAC were reported to be $15-37 \%{ }^{29-35}$. Several studies showed that NRM after RTMAC regimens without TBI contributed to less NRM than that after conventional MAC in adults $(12 \%)^{11,12}$ and in children $(13.6 \%)^{26}$. Our RTMAC regimen induced no NRM. Thus, the reducing dose of TBI at least to 8Gy appears to confer less organ damage on pediatric patients.

(2) Graft failure is one of the major problems for CBT after an NMAC/RIC/RTMAC regimen ${ }^{20,21,26}$. Paillard et al..$^{20}$ and Bradley et al. ${ }^{21}$ reported that 4 of $5 \mathrm{CB}$ and 6 of $21 \mathrm{CB}$, respectively, failed to engraft into pediatric patients, when RIC was used as the preparative conditioning. Satwani et al. ${ }^{26}$ reported that graft failure occurred in 16 of 51 patients who received CBT after non TBI-based RTMAC regimen. In our study, all 9 patients who received CBT achieved engraftment after 8-Gy TBI-based RTMAC regimen excluding one patient with early relapse. Thus, our RTMAC regimen may have sufficient intensity to engraft $\mathrm{CB}$ into patients with pediatric hematological malignancies, resulting in comparable 5-year OS and RFS between CBT and BMT (Table 3).

(3) High NRM is one of the important problems in second allo$\mathrm{HSCT}^{36}$. In our study, 9 patients relapsed hematologically after the first allo-HSCT, but 6 patients could receive a second alloHSCT. Two of them are alive and disease-free. The remaining 4 patients were dead of disease progression. NRM was not observed after second allo-HSCT. Thus, relapsed patients who received a first allo-HSCT after our RTMAC could undergo second allo-HSCT with relative safety.

Satwani et al. ${ }^{26}$ reported the largest study of pediatric patients with malignant disease ( $n=50 ; 24$ patients with leukemia/MDS, 16 patients with lymphoma, 10 patients with neuroblastoma) who had received non TBI-based RTMAC for allo-HSCT. According to their report, 5-year OS and 5-year RFS were 64\% and 58\%, respectively. Our OS and RFS compare favorably with those of theirs, although patients' characteristics differed widely between two groups. Our 8-Gy TBI-based RTMAC regimen would have equal effectiveness to BU-based RTMAC regimens.

In adult ALL cases, there have been few reports of successful outcomes after allo-HSCT prepared with NMAC/RIC, probably due to inadequate GVL effects and high $\mathrm{RRT}^{8,9,10}$. Kato et al. ${ }^{27}$, however, reported that 5 of 7 pediatric patients with ALL who received alloHSCT after RTMAC consisting of FLU, cytarabine, granulocyte colony-stimulating factor and melphalan were alive in CR for a long period of time. In our study, 9 of 11 pediatric patients with ALL in CR are disease-free alive at present Median follow-up period is 6.6 years (range, 2.4-10.1 years). Thus, allo-HSCT combined with RTMAC regimen seems to exert antileukemic effects for children with ALL in CR. 


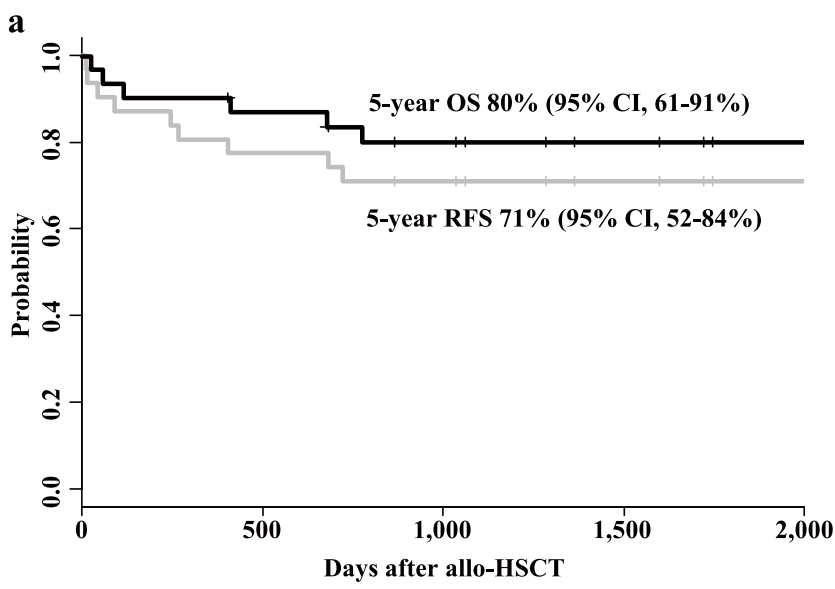

b

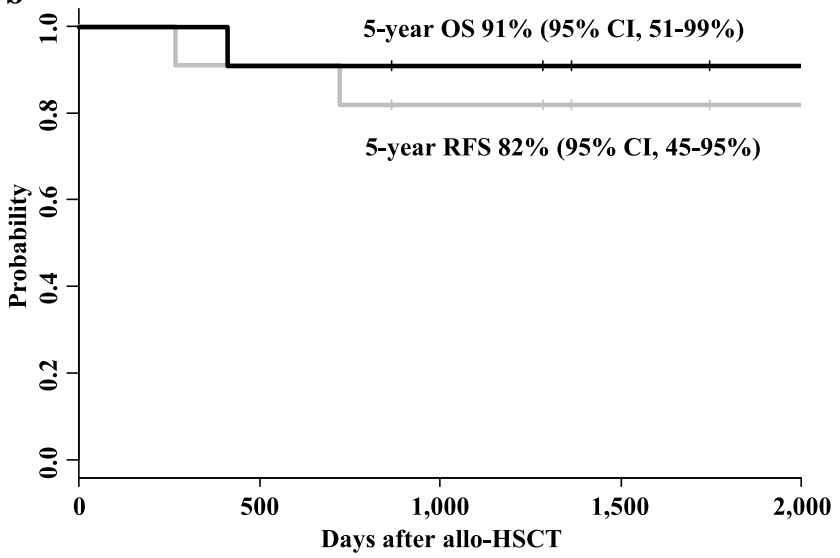

c

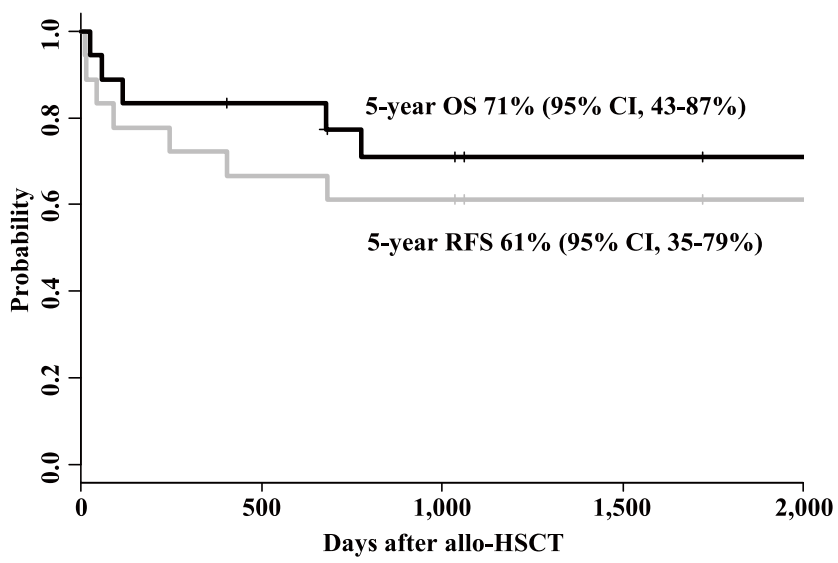

Figure 1 Kaplan-Meier estimates of overall survival (OS) and relapsefree survival (RFS) of patients who received allo-HSCT after an 8-Gy TBI/ FLU/CY conditioning regimen. (a) 5-year OS and RFS for all 31 patients, (b) 5-year OS and RFS for 11 patients with acute lymphoblastic leukemia, (c) 5-year OS and RFS for 18 patients with myeloid malignancies (acute myeloid leukemia, myelodysplastic syndrome or juvenile myelomonocytic leukemia patients).

In conclusion, this study indicates that allo-HSCT with an 8-Gy TBI/FLU/CY RTMAC regimen would be less toxic and has enough ability to engraft for children with hematological malignancies. An evaluation of long term prognosis including late effects will be necessary for this RTMAC regimen.

\section{Methods}

Study design and patient eligibility. A retrospective single-center analysis was performed on patients with leukemia or MDS, aged $\leq 20$ years, who had received an
RTMAC regimen consisting of 8-Gy TBI/FLU/CY followed by allo-HSCT at Shinshu University Hospital between March 2004 and December 2012. Patient information until August 31, 2014 was used in all transplanted children. The following patients were excluded from this study: (1) patients with chromosomal abnormalities in the germline; (2) patients with a history of prior allo-HSCT; (3) patients with a history of radiation therapy. Written informed consent was obtained from each patient and/or guardians before allo-HSCT. The indications for allo-HSCT in initial CR for AML, included high-risk cytogenetics (monosomy 7), morphologic subtypes M6 or M7 and AML with myelodysplasia-related changes. Allo-HSCTs were performed in first CR for ALL patients who had high-risk chromosomal translocations [t $(9 ; 22)$ or MLL rearrangement], had initial induction failure or had the allo-HSCT risk of the Tokyo Children's Cancer Study Group Study L99-15 $5^{37} / \mathrm{L} 04-16$ (Unique trial number: UMIN000001413). All patients with ALAL underwent allo-HSCT. Allo-HSCT was indicated for all relapsed AML and ALL except B-lineage ALL that relapsed 6 months after completion of chemotherapy or isolated extramedullary relapse. Forty-one patients received their first allo-HSCT during study period. Among them, 31 of the patients underwent allo-HSCT after the 8-Gy TBI/FLU/CY regimen. For 7 patients with ALL in non-CR, we used a 12-Gy TBI/FLU/CY regimen instead of RTMAC. A 12-Gy TBI-based regimen or full dose BU-based regimen was applied to 2 patients with ALL in CR and 1 patient with JMML because one of the patient's guardians requested this regimen, and 2 patients (Philadelphia-positive ALL and JMML) were transplanted according to the protocols of the Japanese Pediatric Leukemia/ Lymphoma Study Group (Unique trial number: C000000290, UMIN000005936).

Ethics statement. The institutional review board at Shinshu University School of Medicine approved all study procedures. The study was performed in accordance with approved guidelines. Written informed consent was obtained from each patient and/or guardians. The study was carried out in compliance with the Helsinki Declaration.

Donor selection. HLA antigens for A, B, C, and DRB1 were determined by highresolution DNA typing. The primary criterion for donor selection was HLA compatibility, defined as a 6/6 allele match (at the A, B, and DRB1 loci) or a 5/6 allele match (single-antigen mismatch at the A, B, or DRB1 locus), with a preference for a 6 / 6 match.

Conditioning regimen and additional craniospinal irradiation. Preparative conditioning regimen consisted of TBI (2-Gy/day, day -7 to day -4$),$ FLU ( $30 \mathrm{mg} /$ $\mathrm{m}^{2} /$ day, day -8 to day -4$)$ and $\mathrm{CY}(60 \mathrm{mg} / \mathrm{kg} /$ day, day $-3,-2)$ (Figure 2). No patients received antithymocyte/antilymphocyte globulin. After allo-HSCT, additional craniospinal irradiation (brain, 10-Gy; spinal, 7-Gy) was performed for patients with central nervous system disease.

GVHD prophylaxis. When the stem cell source was an HLA-identical sibling (at the $\mathrm{A}, \mathrm{B}$, and DRB1 loci), short-term methotrexate and cyclosporine A (starting at $3 \mathrm{mg} /$ $\mathrm{kg} /$ day on day -1) were used for acute GVHD prophylaxis. When BM cells were obtained from a mismatched sibling, a parent, or an unrelated donor, we used shortterm methotrexate and tacrolimus (starting at $0.03 \mathrm{mg} / \mathrm{kg} / \mathrm{day}$ on day -1 ) with or without methylprednisolone. In CBT, tacrolimus and methylprednisolone were administered intravenously. Acute and chronic GVHD was graded according to standard criteria ${ }^{38,39}$.

Supportive care. Granulocyte-colony stimulating factor was administered intravenously to ALL patients from day 5 , and its use was discontinued after the neutrophil count exceeded $1,500 / \mu \mathrm{L}$. Acyclovir $(10 \mathrm{mg} / \mathrm{kg} /$ day $)$ and micafungin $(2 \mathrm{mg} / \mathrm{kg} /$ day $)$ were given intravenously during the peritransplant period. Intravenous gamma globulin was administered at $200 \mathrm{mg} / \mathrm{kg}$ every 2 weeks up to day 60 , and thereafter monthly up to 1 year. Trimethoprim/sulfamethoxazole was used after engraftment for prophylaxis of Pneumocystis jiroveci infections.

Assessment of engraftment. Neutrophil engraftment was defined as the first of three consecutive days with an absolute neutrophil count of at least $500 / \mu \mathrm{L}$. Platelet engraftment was defined as the first day that the platelet count exceeded $50,000 / \mu \mathrm{L}$ without platelet transfusion. Engraftment failure was defined by the absence of blood cell count recovery at day 100 or hematopoietic reconstitution with autologous cells. Hematopoietic chimerism was evaluated in BM cells by fluorescent in situ hybridization with the probes specific to sex chromosomes and/or by analysis of DNA microsatellite polymorphisms by polymerase chain reaction $(\mathrm{PCR})^{40}$. Chimerism was assessed at 1,2 and 3 months after allo-HSCT and subsequently every 3 to 6 months for 2 years. Full donor chimerism was defined as the presence of higher than $99 \%$ donor cell profiling.

Definition of CR, relapse, and minimal residual disease (MRD). CR was defined as a normal peripheral blood cell count (absolute neutrophil count $>1,000 / \mu \mathrm{L}$ and platelet count $>100,000 / \mu \mathrm{L}$ ) and normocellular marrow with less than $5 \%$ blasts in the marrow. In addition, no signs or symptoms are evident of central nervous system leukemia or other extramedullary infiltration. Hematological relapse was defined as more than $20 \%$ blasts in the marrow. Hematological relapse within 100 days of alloHSCT was defined as early relapse. In ALL and ALAL, we evaluated MRD by the presence of rearrangements of genes of immunoglobulin heavy chain or T-cell receptor using $\mathrm{PCR}^{41}$ 
Table 3 | Univariate analyses of prognostic factors in 31 patients who received allo-HSCT after 8-Gy TBI/FLU/CY conditioning regimen

\begin{tabular}{|c|c|c|c|c|}
\hline & Percentage 5-year OS (95\% Cl) & $p$-value & Percentage 5-year RFS (95\% Cl) & p-value \\
\hline \multicolumn{5}{|l|}{ Age } \\
\hline$<10$ years & $83(56-94)$ & 0.31 & $78(51-91)$ & 0.38 \\
\hline \multicolumn{5}{|l|}{ Sex } \\
\hline Male & 87 (57-97) & 0.19 & $81(53-94)$ & 0.19 \\
\hline Female & $73(43-89)$ & & $60(32-80)$ & \\
\hline \multicolumn{5}{|l|}{ Stem cell source } \\
\hline BM & $75(50-89)$ & 0.34 & $62(38-79)$ & 0.15 \\
\hline$C B$ & 90 (47-99) & & $90(47-99)$ & \\
\hline Related & 79 (47-93) & 0.68 & $60(32-80)$ & 0.24 \\
\hline Unrelated & $81(53-94)$ & & $81(53-94)$ & \\
\hline \multicolumn{5}{|l|}{ HLA } \\
\hline $\begin{array}{l}\text { 8-7/8 allele matched related } B M \text { or } 8 / 8 \text { allele } \\
\text { matched unrelated } B M / C B\end{array}$ & $84(49-96)$ & 0.98 & $69(37-87)$ & 0.94 \\
\hline Others & $77(50-91)$ & & $72(46-87)$ & \\
\hline \multicolumn{5}{|l|}{ Disease status } \\
\hline CR & 95 (70-99) & $<0.01$ & $81(57-92)$ & 0.03 \\
\hline $\begin{array}{l}\text { Non-CR } \\
\text { Acute GVHD }\end{array}$ & $50(18-75)$ & & $50(18-75)$ & \\
\hline $\begin{array}{l}\text { Acute GVHD } \\
\text { Grade 0, I } \\
\text { Grade II-IV }\end{array}$ & $\begin{array}{l}76(47-90) \\
86(54-96)\end{array}$ & 0.29 & $\begin{array}{l}59(33-78) \\
86(54-96)\end{array}$ & 0.12 \\
\hline \multicolumn{5}{|l|}{ Chronic GVHD } \\
\hline Yes & $73(50-87)$ & 0.09 & $65(42-81)$ & 0.23 \\
\hline No & 100 & & $88(39-98)$ & \\
\hline \multicolumn{5}{|l|}{ Interval from onset to transplantation } \\
\hline $\begin{array}{l}<2 \text { years } \\
\geq 2 \text { years }\end{array}$ & $\begin{array}{l}79(56-91) \\
86(33-98)\end{array}$ & 0.57 & $\begin{array}{l}67(44-82) \\
86(33-98)\end{array}$ & 0.34 \\
\hline
\end{tabular}

BM, bone marrow; $\mathrm{CB}$, cord blood; $\mathrm{Cl}$, confidence interval; $\mathrm{CR}$, complete remission; $\mathrm{CY}$, cyclophosphamide; FLU, fludarabine; GVHD, graft-versus-host disease; $\mathrm{HLA}$, human leukocyte antigen; HSCT, hematopoietic stem cell transplantation; OS, overall survival; RFS, relapse-free survival; TBI, total body irradiation.

\begin{tabular}{|c|c|c|c|c|c|c|c|c|c|c|c|}
\hline & & Day & -8 & -7 & -6 & -5 & -4 & -3 & -2 & -1 & $\mathbf{0}$ \\
\hline Total body irradiation & $2 \mathbf{G y}$ & & & $\downarrow$ & $\downarrow$ & $\downarrow$ & $\downarrow$ & & & & \\
\hline Fludarabine & $30 \mathrm{mg} / \mathrm{m}^{2}$ & & $\downarrow$ & $\downarrow$ & $\downarrow$ & $\downarrow$ & $\downarrow$ & & & & \\
\hline Cyclophosphamide & $60 \mathrm{mg} / \mathrm{kg}$ & & & & & & & $\downarrow$ & $\downarrow$ & & \\
\hline
\end{tabular}

Figure $2 \mid$ Schedule of a reduced-toxicity myeloablative conditioning regimen consisting of 8-Gy total body irradiation, fludarabine and cyclophosphamide.

Assessment of toxicity. RRT during the period from conditioning up to day 100 of allo-HSCT was assessed according to the National Cancer Institute's Common Terminology Criteria for Adverse Events Version 4.0. All transplant events excluding GVHD and hematological toxicities were categorized as RRT. Death after relapse was categorized as death due to the disease progression irrespective of any other causes. Deaths in the absence of active malignancy were categorized as NRM.

Statistical analyses. Actuarial estimates of OS and RFS were made using the KaplanMeier technique. Patients were censored for OS if they were alive at last follow-up. Patients were censored for RFS if they showed continued CR after first allo-HSCT. We used a logrank test to evaluate prognostic factors for 5-year OS and PFS. We calculated the parameters including disease status (CR vs. non-CR), age ( $<10$ years of age vs. $\geq 10$ years), sex, donor type (BM vs. CB, related vs. unrelated), HLA (8-7/8 allele matched related $\mathrm{BM}$ or $8 / 8$ matched unrelated $\mathrm{BM} / \mathrm{CB}$ vs. others), GVHD and interval from onset to transplantation ( $<2$ years vs. $\geq 2$ years). Analysis was performed with $\mathrm{EZR}^{42}$.

1. Hahn, T. et al. The role of cytotoxic therapy with hematopoietic stem cell transplantation in the therapy of acute lymphoblastic leukemia in children: an evidence-based review. Biol Blood Marrow Transplant. 11, 823-861 (2005).

2. Oliansky, D. M. et al. The role of cytotoxic therapy with hematopoietic stem cell transplantation in the therapy of acute myeloid leukemia in children: an evidencebased review. Biol Blood Marrow Transplant. 13, 1-25 (2007).
3. Appelbaum, F. R. Haematopoietic cell transplantation as immunotherapy. Nature 411, 385-389 (2001).

4. Satwani, P. et al. Reduced intensity and non-myeloablative allogeneic stem cell transplantation in children and adolescents with malignant and non-malignant diseases. Pediatr Blood Cancer. 50, 1-8 (2008).

5. Bearman, S. I. et al. Regimen-related toxicity in patients undergoing bone marrow transplantation. J Clin Oncol. 6, 1562-1568 (1988)

6. Clift, R. A. et al. Allogeneic marrow transplantation in patients with acute myeloid leukemia in first remission: a randomized trial of two irradiation regimens. Blood 76, 1867-1871 (1990).

7. Giralt, S. et al. Engraftment of allogeneic hematopoietic progenitor cells with purine analog-containing chemotherapy: harnessing graft-versus-leukemia without myeloablative therapy. Blood 89, 4531-4536 (1997).

8. Arnold, R. et al. Nonmyeloablative stem cell transplantation in adults with highrisk ALL may be effective in early but not in advanced disease. Leukemia 16, 2423-2428 (2002).

9. Martino, R. et al. Allogeneic hematopoietic stem cell transplantation with reduced-intensity conditioning in acute lymphoblastic leukemia: a feasibility study. Haematologica 88, 555-560 (2003).

10. Hamaki, T. et al. Reduced-intensity stem-cell transplantation for adult acute lymphoblastic leukemia: a retrospective study of 33 patients. Bone Marrow Transplant. 35, 549-356 (2005).

11. Andersson, B. S. et al. Once daily i.v. busulfan and fludarabine (i.v. Bu-Flu) compares favorably with i.v. busulfan and cyclophosphamide (i.v. BuCy2) as 
pretransplant conditioning therapy in AML/MDS. Biol Blood Marrow Transplant. 14, 672-684 (2008).

12. Bredeson, C. N. et al. Outcomes following HSCT using fludarabine, busulfan, and thymoglobulin: a matched comparison to allogeneic transplants conditioned with busulfan and cyclophosphamide. Biol Blood Marrow Transplant. 14, 993-1003 (2008).

13. Bresters, D. et al. High burden of late effects after haematopoietic stem cell transplantation in childhood: a single-centre study. Bone Marrow Transplant. 45, 79-85 (2010).

14. Ferry, C. et al. Long-term outcomes after allogeneic stem cell transplantation for children with hematological malignancies. Bone Marrow Transplant. 40, 219-224 (2007).

15. Leiper, A. D. Non-endocrine late complications of bone marrow transplantation in childhood: part I. Br J Haematol. 118, 3-22 (2002).

16. Leiper, A. D. Non-endocrine late complications of bone marrow transplantation in childhood: part II. Br J Haematol. 118, 23-43 (2002).

17. Brennan, B. M. \& Shalet, S. M. Endocrine late effects after bone marrow transplant. Br J Haematol. 118, 58-66 (2002).

18. Yanagisawa, R. et al. Low toxicity of a conditioning with 8-Gy total body irradiation, fludarabine and cyclophosphamide as preparative regimen for allogeneic hematopoietic stem cell transplantation in pediatric hematologica malignancies. Pediatr Transplant. 13, 737-745 (2009).

19. Pulsipher, M. A. et al. Reduced-intensity allogeneic transplantation in pediatric patients ineligible for myeloablative therapy: results of the Pediatric Blood and Marrow Transplant Consortium Study ONC0313. Blood 114, 1429-1436 (2009).

20. Paillard, C. et al. Reduced-intensity conditioning followed by allogeneic transplantation in pediatric malignancies: a report from the Société Française des Cancers de l'Enfant and the Société Française de Greffe de Moelle et de Thérapie Cellulaire. Bone Marrow Transplant. 48, 1401-1408 (2013).

21. Bradley, M. B. et al. Reduced intensity allogeneic umbilical cord blood transplantation in children and adolescent recipients with malignant and nonmalignant diseases. Bone Marrow Transplant. 40, 621-631 (2007).

22. Del Toro, G. et al. A pilot study of reduced intensity conditioning and allogeneic stem cell transplantation from unrelated cord blood and matched family donors in children and adolescent recipients. Bone Marrow Transplant. 33, 613-622 (2004).

23. Strullu, M. et al. Allogeneic hematopoietic stem cell transplantation following reduced-intensity conditioning regimen in children: a single-center experience. Eur J Haematol. 88, 504-509 (2012).

24. Verneris, M. R. et al. Reduced-intensity conditioning regimens for allogeneic transplantation in children with acute lymphoblastic leukemia. Biol Blood Marrow Transplant. 16, 1237-1244 (2010).

25. Law, J. et al. Busulfan, fludarabine, and alemtuzumab as a reduced toxicity regimen for children with malignant and nonmalignant diseases improves engraftment and graft-versus-host disease without delaying immune reconstitution. Biol Blood Marrow Transplant. 18, 1656-1663 (2012).

26. Satwani, P. et al. Transplantation-related mortality, graft failure, and survival after reduced-toxicity conditioning and allogeneic hematopoietic stem cell transplantation in 100 consecutive pediatric recipients. Biol Blood Marrow Transplant. 19, 552-561 (2013).

27. Kato, K., Yoshida, N., Matsumoto, K. \& Matsuyama, T. Fludarabine, cytarabine, granulocyte colony-stimulating factor and melphalan (FALG with L-PAM) as a reduced toxicity conditioning regimen in children with acute leukemia. Pediatr Blood Cancer. 61, 712-716 (2014).

28. Petropoulos, D. et al. Total body irradiation, fludarabine, melphalan, and allogeneic hematopoietic stem cell transplantation for advanced pediatric hematologic malignancies. Bone Marrow Transplant. 37, 463-467 (2006).

29. Leung, W. et al. High success rate of hematopoietic cell transplantation regardless of donor source in children with very high-risk leukemia. Blood 118, 223-230 (2011)

30. Strahm, B. et al. Hematopoietic stem cell transplantation for advanced myelodysplastic syndrome in children: results of the EWOG-MDS 98 study. Leukemia 25, 455-462 (2011).
31. Fagioli, F. et al. Hematopoietic stem cell transplantation for children with highrisk acute lymphoblastic leukemia in first complete remission: a report from the AIEOP registry. Haematologica 98, 1273-1281 (2013).

32. Sisler, I. Y. et al. Impact of conditioning regimen in allogeneic hematopoetic stem cell transplantation for children with acute myelogenous leukemia beyond first complete remission: a pediatric blood and marrow transplant consortium (PBMTC) study. Biol Blood Marrow Transplant. 15, 1620-1627 (2009).

33. Horan, J. T. et al. Impact of disease risk on efficacy of matched related bone marrow transplantation for pediatric acute myeloid leukemia: the Children's Oncology Group. J Clin Oncol. 26, 5797-5801 (2008).

34. Klingebiel, T. et al. Results and factors influencing outcome after fully haploidentical hematopoietic stem cell transplantation in children with very highrisk acute lymphoblastic leukemia: impact of center size: an analysis on behalf of the Acute Leukemia and Pediatric Disease Working Parties of the European Blood and Marrow Transplant group. Blood 115, 3437-3446 (2010).

35. Dini, G. et al. No difference in outcome between children and adolescents transplanted for acute lymphoblastic leukemia in second remission. Blood 118, 6683-6690 (2011).

36. Fathi, A. T. \& Chen, Y. B. Treatment of relapse of acute myeloid leukemia after allogeneic hematopoietic stem cell transplantation. Curr Hematol Malig Rep. 9, 186-192 (2014).

37. Manabe, A. et al. Significance of the complete clearance of peripheral blasts after 7 days of prednisolone treatment in children with acute lymphoblastic leukemia: the Tokyo Children's Cancer Study Group Study L99-15. Haematologica 93, 1155-1160 (2008)

38. Przepiorka, D. et al. 1994 Consensus Conference on Acute GVHD Grading. Bone Marrow Transplant. 15, 825-828 (1995).

39. Filipovich, A. H. et al. National Institutes of Health consensus development project on criteria for clinical trials in chronic graft-versus-host disease: I. Diagnosis and staging working group report. Biol Blood Marrow Transplant. 11, 945-956 (2005).

40. Matsuda, K. et al. Monitoring of hematopoietic chimerism by short tandem repeats, and the effect of CD selection on its sensitivity. Clin Chem. 50, 2411-2414 (2004).

41. Matsuda, K., Sugano, M. \& Honda, T. PCR for monitoring of minimal residual disease in hematologic malignancy. Clin Chim Acta. 18, 74-80 (2012).

42. Kanda, Y. Investigation of the freely available easy-to-use software 'EZR' for medical statistics. Bone Marrow Transplant. 48, 452-458 (2013).

\section{Author contributions}

K.H., Y.N. and K.K. wrote the main manuscript. K.S., Y.N. and K.K. conceived the conditioning regimen. T.K., S.S., K.Y., M.T. and R.Y. contributed to material of the research. All authors reviewed the manuscript.

\section{Additional information}

Competing financial interests: The authors declare no competing financial interests.

How to cite this article: Hirabayashi, K. et al. Reduced-toxicity myeloablative conditioning consisting of 8-Gy total body irradiation, cyclophosphamide and fludarabine for pediatric hematological malignancies. Sci. Rep. 4, 6942; DOI:10.1038/srep06942 (2014).

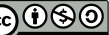

This work is licensed under a Creative Commons Attribution-NonCommercialShareAlike 4.0 International License. The images or other third party material in this article are included in the article's Creative Commons license, unless indicated otherwise in the credit line; if the material is not included under the Creative Commons license, users will need to obtain permission from the license holder in order to reproduce the material. To view a copy of this license, visit http:// creativecommons.org/licenses/by-nc-sa/4.0/ 